Pesq. Vet. Bras. 36(7):611-616, julho 2016 DOI: $10.1590 /$ S0100-736X2016000700009

\title{
Avaliação da composição molecular da cápsula anterior da lente de cães idosos com catarata de alto risco ${ }^{1}$
}

\author{
Ana Paula Hvenegaard², Paulo S.M. Barros², Angélica M.V. Safatle ${ }^{2}$, Ana Carolina \\ A. Góes ${ }^{2}$, Ana R. Eyherabide ${ }^{2}$ e Nadia C.O. Miguel $^{3}$
}

\begin{abstract}
Hvenegaard A.P., Barros P.S.M., Safatle A.M.V., Góes A.C.A., Eyherabide A.R. \& Miguel N.C.O. 2016. [Molecular evaluation of the anterior lens capsule of older dogs suffering from high risk cataracts.] Avaliação da composição molecular da cápsula anterior da lente de cães idosos com catarata de alto risco. Pesquisa Veterinária Brasileira 36(7):611-616. Serviço de Oftalmologia, Faculdade de Medicina Veterinária e Zootecnia, Universidade de São Paulo, Av. Prof. Orlando Marques de Paiva 87, Cidade Universitária, São Paulo, SP 05508-270, Brazil. E-mail: ana6113@usp.br

Nowadays, the anterior lens capsule and its epithelium have been being frequently studied aiming to reduce the incidence of posterior lens capsule opacity, a complication that frequently occurs after surgical removal of cataracts, due to epithelium cells differentiation and migration to the posterior pole. The objective of this study was to evaluate by histochemistry (PAS and picrosirius red) analysis two important molecular components of the anterior lens capsule (proteoglycans and type IV collagen) in older diabetic and non-diabetic dogs, with diabetic and hypermature cataracts, after phacoemulsification surgery utilizing $0.1 \%$ trypan blue or not. Twenty seven dogs, including 21 female and 6 male dogs, with ages varying from 8 to 12 years old (mean $=9.6$ yo) of different breeds were studied. The animals were divided into 2 groups: GC (hypermature cataracts) and GCD (diabetic cataracts). Results showed that, besides their different pathophysiologies, both types of capsules studied (diabetic and hypermature ones) presented the same molecular composition of proteoglycans and type IV collagen, since no statistical significant differences were observed. In addition, $0.1 \%$ trypan blue was not capable to induce any other evident alteration for the samples. In conclusion, our findings suggest that, if the results consist in alteration induced by the aggressive metabolism of the diabetic cataract or the chronicity of the hypermature one, it is of the same intensity and independent of the use of $0.1 \%$ trypan blue. It is also possible to suggest that this alteration must be capable to compromise lens epithelium cell metabolism, which should probably favour future lens posterior capsule studies.
\end{abstract}

INDEX TERMS: Lens, dogs, diabetes, Trypan blue, cataracts.

RESUMO.- Atualmente, a cápsula anterior e o epitélio da lente tem sido cada vez mais estudados, com o intuito de reduzir as possíveis complicações do pós-operatório da remoção da catarata, tal como a opacidade da cápsula pos-

\footnotetext{
${ }^{1}$ Recebido em 25 de novembro de 2015.

Aceito para publicação em 2 de fevereiro de 2016.

${ }^{2}$ Serviço de Oftalmologia, Faculdade de Medicina Veterinária e Zootecnia, Universidade de São Paulo (USP), Av. Prof. Orlando Marques de Paiva 87, Cidade Universitária, São Paulo, SP 05508-270, Brasil. Pesquisa de doutorado com apoio CAPES. *Autor para correspondência: ana6113@usp.br

${ }^{3}$ Programa de Graduação em Histologia, Programa de Pesquisa em Biologia Celular e do Desenvolvimento, Instituto de Ciências Biomédicas (ICB), Centro de Ciência da Saúde (CCS), Universidade Federal do Rio de Janeiro (UFRJ), Ilha do Fundão, Av. Carlos Chagas Filho 373, Bloco F2-008 e F2-012, Rio de Janeiro, RJ 21044-020, Brasil.
}

terior, alteração ocasionada principalmente pela diferenciação e migração das células do epitélio lenticular para a cápsula posterior da lente. 0 objetivo deste estudo foi analisar a composição molecular da cápsula anterior da lente pela técnica histoquímica de PAS (avaliação de proteoglicanos) e picrosirius red (avaliação de colágeno IV), em cães idosos com catarata diabética e não diabética do tipo hipermadura, submetidos ao uso ou não de azul de tripano a 0,1 $\%$ durante a facoemulsificação. Vinte e sete cães foram estudados, incluindo 21 fêmeas e 6 machos, de 8 a 12 anos de idade (média = 9,6 anos), de diversas raças e divididos em 2 grupos: GC (catarata hipermadura) e GCD (catarata diabética). Os resultados das análises realizadas mostraram que ambas as amostras, tanto as provenientes das cataratas hi- 
permaduras, quanto das diabéticas, apresentam semelhante composição molecular de proteoglicanos e colágeno IV e isto independente da utilização de azul de tripano a 0,1 $\%$. Conclui-se, portanto, que se os resultados obtidos forem decorrentes de alterações provocadas pelo rápido metabolismo da catarata diabética e pela cronicidade da catarata hipermadura sugere-se que o comprometimento da estrutura capsular seja de intensidade equivalente e, por consequência, que isto também possa prejudicar o metabolismo das células do epitélio anterior da lente, diminuindo assim a incidência da opacidade da cápsula posterior de cães com catarata diabética e hipermadura submetidos à facoemulsificação.

TERMOS DE INDEXAÇÃO: Lente, cães, diabetes, Azul de tripano, catarata.

\section{INTRODUÇÃO}

A cápsula anterior da lente e suas células epiteliais vem sendo estudadas há bastante tempo por serem consideradas as principais responsáveis pela formação da opacidade de cápsula posterior (Fig.1), uma das principais complicações observadas no pós-cirúrgico desta intervenção. (Vaughan et al. 2003, Mohamad et al. 2005, Nanavaty et al. 2006, Sigle \& Nassisse 2006, Portes et al. 2007, Portes et al. 2010, Kim et al. 2012, Jung et al. 2013).

A cápsula anterior da lente é a lâmina basal do epitélio invertido (Winkler et al. 2001), ou seja, uma matriz extracelular especializada, que desempenha papel fundamental na adesão e diferenciação celular, na permeabilidade e filtração de substâncias, na polaridade epitelial e no crescimento celular das fibras lenticulares (Lebleu et al. 2007, Halfter et al. 2013). É constituída principalmente por interações entre proteoglicanos, fibras colágenas (IV e XVIII) e glicoproteínas de adesão (Yan et al. 2005, Lebleu et al. 2007, Danysh \& Duncan 2009, Nelson \& Cox 2011, Halfter et al. 2013).

Os proteoglicanos (perlecan, nidogênio/entactina, fi-

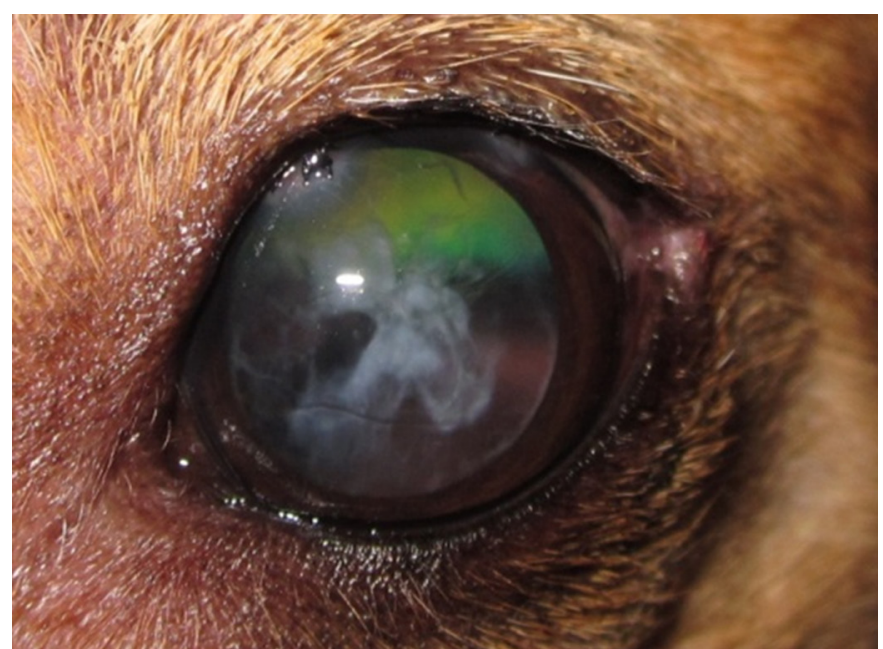

Fig.1. Opacidade de cápsula posterior observada em olho esquerdo sob midríase medicamentosa, em um cão macho, 7 anos, sem raça definida, após 3 meses da FACO. Observar a possível visibilização do fundo de olho (tapetum - coloração amarelo-esverdeada e região não-tapetal - marrom-avermelhada). bronectina e a laminina), são os principais componentes de todas as lâminas basais dos seres vivos. São caracterizados por macromoléculas formadas por uma ou mais cadeias de glicosaminoglicanos sulfatados da matriz ou da superfície celular, ligados ao ácido hialurônico, covalentemente unidos a uma proteína de membrana ou a uma proteína secretada (Voet et al. 2008, Nelson \& Cox 2011). Além de ser parte fundamental da estrutura da lente, os proteoglicanos são também responsáveis pelo controle de diversas atividades celulares, tais como a ativação de fatores de crescimento, a migração e adesão celular e a propagação de "informações" através de suas membranas (Smyth et al. 1999, Yan et al. 2005, Lebleu et al. 2007, Danysh \& Duncan 2009, Nelson \& Cox 2011, Halfter et al. 2013). O colágeno tipo IV, por sua vez, é uma proteína não fibrilar estável que compõe aproximadamente $50-80 \%$ da estrutura da cápsula. Integrado à laminina, é responsável pela manutenção da forma da lente durante seu rápido desenvolvimento; por sua estabilidade e por sua resistência no que diz respeito às forças exercidas pelo corpo ciliar durante o fenômeno de acomodação (Yan et al. 2005, Lebleu et al. 2007, Danysh \& Duncan 2009, Halfter et al. 2013); além disso, o colágeno é também responsável por manter o gradiente aniônico dentro da lente, já que, ligado ao heparansulfato e aos grupos carboxila, forma uma "tela de filtração" que auxilia no controle da difusão e no transporte das moléculas através da lente (Winkler et al. 2001).

Por outro lado, a cápsula é um tecido biológico que sofre alterações significativas na presença de doenças e/ou com o envelhecimento (Bernays \& Peiffer 2000, Winkler et al. 2001, Krag \& Andreassen 2003, Barros et al. 2008, Danysh \& Duncan 2009). A espessura capsular aumenta tanto com a idade (Bernays \& Peiffer 2000, Danysh \& Duncan 2009), quanto na presença de diabetes (Barros et al. 2008). Além disso, observa-se a diminuição da taxa de difusão molecular através da cápsula (Danysh \& Duncan 2009), a perda da quantidade e do arranjo das moléculas estruturais (Winkler et al. 2001), a perda de suas propriedades materiais e estruturais (Krag \& Andreassen 2003), da elasticidade e de sua força mecânica (Marshall et al. 1992, Bailey et al. 1993) e a diminuição na composição de água (Yang et al. 1998).

A catarata é uma doença oftálmica frequentemente diagnosticada em cães (Martin 2005) que também pode acometer a cápsula lenticular (Davidson \& Nelms 2007). Classificada de acordo com seu estágio de desenvolvimento, etiologia, localização, idade do surgimento e/ou consistência (Slatter 2005, Ofri 2008), apresenta dois tipos que mais se destacam pela gravidade das alterações que provoca e pelo alto risco de inflamação intraocular: a catarata diabética, do tipo intumescente, de evolução rápida e metabolismo agressivo, com acometimento bilateral e independente da administração da insulina (Beam et al. 1999, Safatle et al. 2000, Slatter 2005) e a do tipo hipermadura, espontânea e genética, de evolução lenta (crônica e tardia), que pode até apresentar-se em início de reabsorção e/ou liquefação (morganiana) (Slatter 2005).

0 tratamento da catarata é cirúrgico e o azul de tripano é o corante mais utilizado (Wollensak et al. 2003, Ozturk \& Osher 2006, Dick et al. 2008) durante o transoperatório, 
antes da capsulorrexe (Basher \& Roberts 1995, Glover \& Constantinescu 1997, Gelatt \& Gelatt 2001, Slatter 2005). Estudos em humanos e coelhos demonstraram que a utilização do azul de tripano não apresenta qualquer toxicidade para o segmento anterior do bulbo ocular (Chang et al. 2005). Entretanto, observou-se que $0,1 \mathrm{ml}$ do corante a $0,0125 \%$, quando aplicado durante 30 segundos sobre a cápsula anterior do cristalino, é capaz de diminuir a densidade e viabilidade das células epiteliais da lente (Nanavaty et al. 2006), podendo até induzir a sua morte em concentrações maiores, como a 0,1\% (Portes et al. 2010).

Não foram encontrados estudos avaliando ou comparando a composição molecular da cápsula anterior da lente de cães com cataratas de diferentes etiologias, estágios de desenvolvimento, idade do surgimento e utilizando-se ou não do azul de tripano a $0,1 \%$ durante a cirurgia. Portanto, o presente estudo teve como principal objetivo comparar a composição de proteoglicanos e colágeno IV, na cápsula anterior da lente de cães idosos, com cataratas de etiologias completamente diferentes (diabética e a hipermadura), mas bastante agressivas, submetidos a remoção cirúrgica da catarata, utilizando-se ou não do azul de tripano a $0,1 \%$ antes da capsulorrexe.

\section{MATERIAL E MÉTODOS}

Amostras. 0 projeto foi aprovado pela Comissão de Ética no uso de animais, da Faculdade de Medicina Veterinária e Zootecnia da Universidade de São Paulo (FMVZ-USP), sob número de protocolo 2964/2013.

Foram incluídos no estudo cães com catarata, diabéticos e não diabéticos, machos ou fêmeas, de 8 a 12 anos. Os cães não diabéticos apresentaram estritamente catarata do tipo hipermadura no olho estudado e, os diabéticos, apenas catarata intumescente decorrente a doença.

Como critérios de exclusão, estabeleceram-se o histórico de trauma, uveítes (agudas ou crônicas) e/ou glaucoma no olho a ser operado; qualquer outro tipo de catarata que não a hipermadura e intumescente secundária a diabete; a presença de quaisquer outras doenças, tanto oftálmicas quanto sistêmicas e a administração de medicações, tanto tópicas quanto sistêmicas, que pudessem comprometer o processo de formação das cataratas ou causar alterações estruturais à cápsula anterior da lente e/ou ao seu epitélio.

Foram selecionados 27 cães, sendo 21 fêmeas e 6 machos, com média de idade de 9,6 anos (8 a 12 anos), de diferentes raças (7 Poodles, 7 Sem Raça Definida, 2 Labradores, 2 Malteses, 2 Schnauzers, 1 Teckel, 1 Beagle, 1 Border Collie, 1 Fox Terrier, 1 Pointer, 1 West Highland White Terrier e 1 Yorkshire Terrier), que foram divididos em dois grupos: GCD (grupo dos cães diabéticos com cataratas diabéticas), incluindo 15 animais (12 fêmeas e 3 machos), apresentando diagnóstico de diabete há 1 até 24 meses (média de 8,2 meses) e catarata intumescente, súbita e bilateral com 1 a 10 meses de evolução (média de 4,7 meses) até o dia da colheita da amostra; e GC (grupo dos cães não diabéticos com cataratas hipermaduras), incluindo 12 animais (9 fêmeas e 3 machos), com histórico de catarata diagnosticada há 4 até 26 meses de evolução (média de 14,6 meses).

Todas as cápsulas anteriores estudadas foram colhidas pelo mesmo cirurgião (A.M.V.S.), após capsulorrexe, com ou sem o uso de azul de tripano $0,1 \%$ (deixado em contato com a cápsula pelo intervalo de tempo de 1 minuto), durante o transoperatório da cirurgia de remoção de catarata por facoemulsificação (FACO). As amostras submetidas ao corante somaram 9 cápsulas de GCD e 5 de GC. Após a capsulorrexe, as amostras foram imediatamente fixadas em formaldeído 10\% e enviadas para laboratórios especializados (Laboratório Pró-biópsia de Histologia Aplicada, São Paulo/SP, Brasil e/ou Laboratório de Neurodegeneração e Reparo do Programa de Pesquisa em Biologia Celular e do Desenvolvimento, Instituto de Ciências Biomédicas, Universidade Federal do Rio de Janeiro). As amostras foram primeiramente desidratadas em concentrações crescentes de etanol, clarificadas em xilol e então incluídas em parafina. Secções transversais de $5 \mu \mathrm{m}$ de espessura, foram obtidas e colocadas em lâminas preparadas com poli-L-lisina para a realização das colorações das técnicas histoquímicas. As amostras foram divididas em: 15 amostras do GC e 12 do GCD para o estudo com ácido periódico de Schiff (PAS), para a avaliação das quantidades de proteoglicanos e 5 amostras de cada grupo (GC e GCD) para o estudo com picrosirius red para a quantidade de colágeno IV. Controles negativos e positivos para as reações também foram preparados.

PAS. Para a coloração com PAS, as lâminas foram primeiramente incluídas em ácido periódico a $0,5 \%$ por $15 \mathrm{~min}$. Foram lavadas em água destilada por 5 minutos e imersas em reagente de Schiff por 30 minutos. Depois, lavagem em água corrente foi realizada por 5 minutos. Para finalizar, realizou-se a imersão das amostras em hematoxilina por 25 segundos, removendo-se o excesso do corante com água destilada. Desidratação, diafanização e montagem com resina finalizaram o processo.

Picrosirius Red. Para a coloração com picrosirius red as lâminas estudadas foram colocadas em solução de ácido fosfomolíbdico por 1 min e coradas durante 90 min em solução de picrosirius (Sirius Red F 3B 200, Mobay Chemical Co. Union, NJ, EUA). Foram lavadas com ácido clorídrico 0,01 normais $(\mathrm{N})$ por 2 min e então lavadas em álcool $70 \%$ por 45 segundos. Os cortes foram desidratados em soluções crescentes de álcool e clarificados com xileno por 5 min e montadas com bálsamo do Canadá.

Captura de imagens e análise estatística. As lâminas preparadas com PAS foram fotomicrografadas no Laboratório de Oncologia Experimental e Comparada (FMVZ-USP), utilizando-se de programa específico para captura de imagens (Image-pro plus, Media Cybernetics, Inc., Rockville, MD, EUA), sob microscópio óptico com aumento de 10 vezes, (Nikon Eclipse E 800, Nikon, EUA com câmera fotográfica Nikon FDX-35 acoplada ao sistema).

As lâminas coradas com picrosirius red, foram inicialmente fotomicrografadas no Laboratório de Neurodegeneração e Reparo do Programa de Pesquisa em Biologia Celular e do Desenvolvimento (ICB-UFRJ), sob microscópio convencional de fluorescência (Zeiss Axioskop 2 plus, Carl Zeiss, Baltimore, Maryland, EUA). Depois, foram observadas sob um microscópio de varredura por laser (LSM) (Zeiss META 510, Carl Zeiss, Baltimore, Maryland, EUA), a 543 nanômetros (nm) de comprimento de onda de excitação para o fluorocromo. As imagens foram adquiridas com resolução de $512 \times 512$ pixels, com $75 \%$ de transmitância a laser, cortes ópticos de 0,7 milimols (mM) de profundidade e 1 min de tempo de varredura no mesmo valor do ganho do detector.

Todas as fotomicrografias foram utilizadas para a realização das mensurações e análises estatísticas comparativas, utilizando-se o programa GraphPad Prism 5 (GraphPad Software Inc., San Diego, CA, EUA) e o teste não paramétrico de Mann-Whitney para o reconhecimento de intensidade de colorações e realização de sua mensuração por densidade óptica. Para tanto, todas as imagens capturadas foram armazenadas em 8-bits, o que permitiu a padronização da escala de semitons de 0 a 255 (escala de densidade óptica - D.O.), sendo “0”, o valor estabelecido para a ausência de pigmentos (preto).

As variáveis também foram descritivamente analisadas em relação ao valor mínimo de 95\% de intervalo de confiança ( $p<0,05)$. 


\section{RESULTADOS}

A coloração em PAS foi capaz de evidenciar a presença dos proteoglicanos nas amostras dos diferentes grupos (Fig.2A e B). Entretanto, não foi observada diferença estatisticamente significante na avaliação da D.O. da coloração das cápsulas estudadas (Quadro 1 e Fig.3).

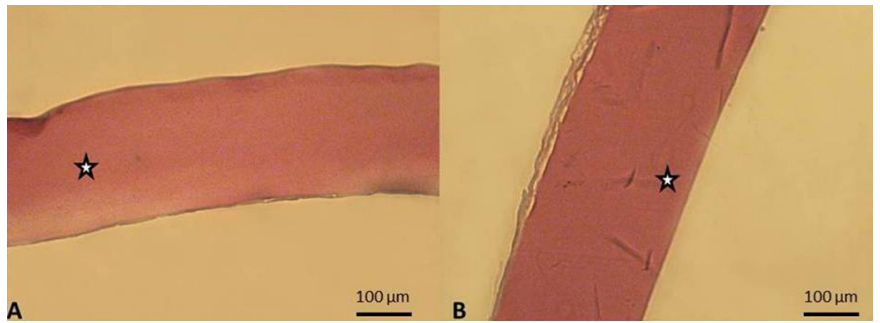

Fig.2. Amostras submetidas à técnica de PAS (A) em um cão da raça West Highland White Terrier de 10 anos do GC, e (B) um cão da raça Maltes de 11 anos do GCD. Observa-se a cápsula (estrela) corada na cor magenta (PAS), evidenciando os proteoglicanos.

Quadro 1. Distribuição dos valores de densidade óptica quanto a coloração de PAS entre os grupos GC e GCD

\begin{tabular}{lccccc}
\hline & Mínima & Mediana & Máxima & Erro Padrão & $\mathrm{P}<0,05$ \\
\hline GC & 96,95 & 129,4 & 169,3 & 8,11 & $\mathrm{P}=0,2176$ \\
GCD & 116,5 & 144,8 & 168,4 & 6,21 &
\end{tabular}

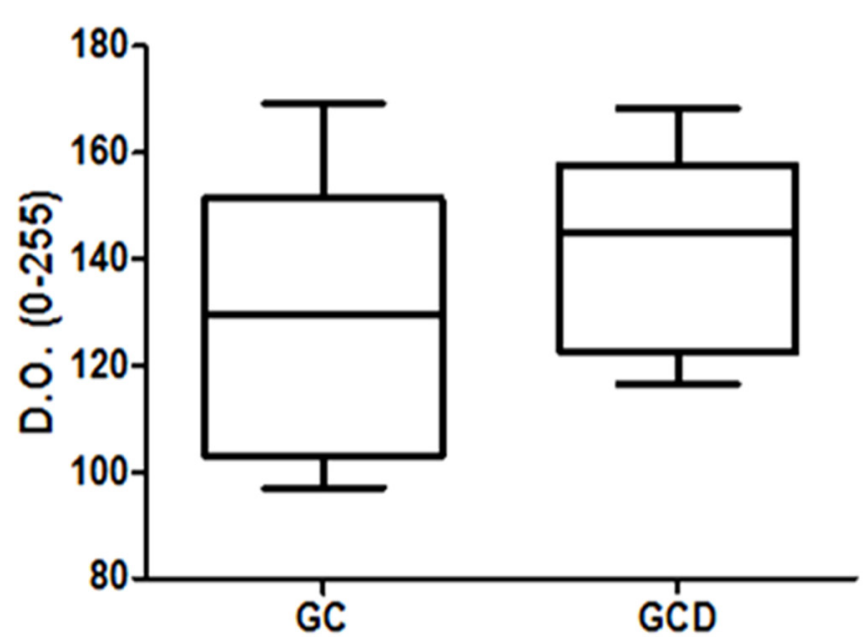

Fig.3. Representação gráfica da análise da densidade óptica da técnica de PAS corado entre os grupos GC e GCD.
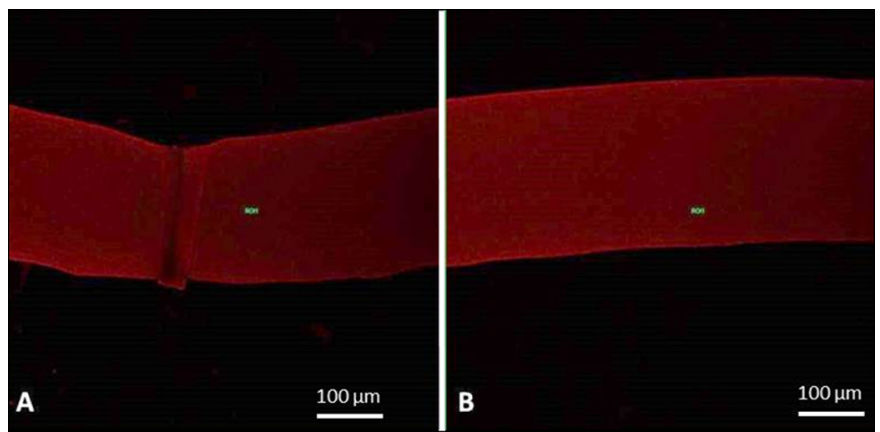

Fig.4. Amostras submetidas ao PSR (A)em um cão da raça Maltes de 11 anos do GC, e (B) em um cão da raça West Highland White Terrier de 10 anos do GCD. Observa-se a cápsula corada na cor vermelha (PSR) evidenciando o colágeno IV.
A coloração em picrosirius red evidenciou a presença de colágeno IV nas amostras (Fig.4 A e B), mas também não demonstrou diferença significativamente relevante quanto a D.O. das colorações das cápsulas dos diferentes grupos (Quadro 2 e Fig.5).

Quadro 2. Distribuição dos valores de densidade óptica quanto a coloração de PSR entre os grupos GC e GCD

\begin{tabular}{lccccc}
\hline & Mínima & Mediana & Máxima & Erro Padrão & $\mathrm{P}<0,05$ \\
\hline GC & 47,13 & 61,25 & 92,16 & 5,93 & $\mathrm{P}=0,1728$ \\
GCD & 10,74 & 45,05 & 87,33 & 8,74 &
\end{tabular}

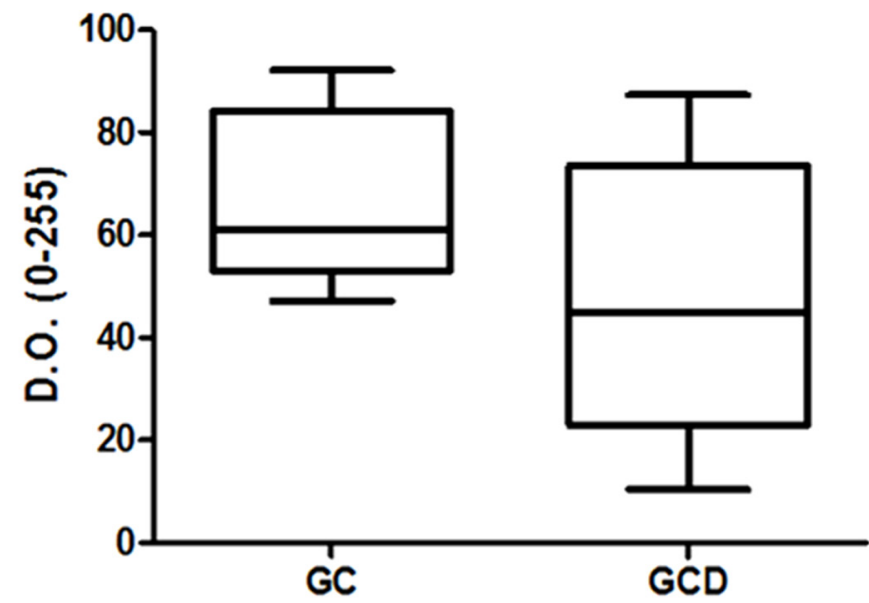

Fig.5. Representação gráfica da análise da densidade óptica da técnica de PSR corado entre os grupos GC e GCD.

\section{DISCUSSÃO}

A composição molecular das cápsulas anteriores de lentes colhidas de dois tipos de cataratas com fisiopatologias completamente diferentes, mas consideradas graves e de alto risco, foram estudadas: a catarata diabética, do tipo intumescente, de evolução rápida e acometimento bilateral (Beam et al. 1999, Safatle et al. 2000, Slatter 2005) e a catarata hipermadura, de evolução lenta, progressiva e crônica (tardia) (Slatter 2005, Ofri 2008, Venkatesh et al. 2010).

Os resultados das análises realizadas neste estudo sugerem que a agressividade do metabolismo que ocorre na lente durante a diabete e a cronicidade de uma catarata com 3 vezes mais tempo de evolução, como a hipermadura, sejam ou capazes de alterar a composição molecular da cápsula de maneira equivalente, ou não influam de maneira significativa, já que ambos os grupos apresentaram cápsulas com a mesma composição molecular de proteoglicanos e colágeno IV. Se os resultados obtidos neste estudo for uma alteração, pode-se ainda sugerir que a correta fisiologia da cápsula e, por consequência, o metabolismo das células de seu epitélio anterior estejam comprometidas (Lebleu et al. 2007, Halfter et al. 2013), o que supostamente diminuiria a incidência de opacidade da cápsula posterior de cães com cataratas deste tipo.

O uso do azul de tripano não foi capaz de causar alterações evidenciáveis na composição molecular das cápsulas, independente dos grupos a que pertenciam; sugerindo, que sua toxicidade seja limitada apenas as células de seu epité- 
lio anterior, como já observado em estudos anteriormente realizados (Nanavaty et al. 2006, Portes et al. 2010, Kim et al. 2012, Jung et al. 2013, Mohamad et al. 2005).

As alterações estruturais ocasionadas na lente por ambos os processos de formação destes tipos de cataratas e a uveíte facoinduzida, inflamação intraocular que pode ocorrer em qualquer momento durante seu curso de evolução, também poderiam deixar seqüelas importantes na morfologia e composição molecular da lente (Basher \& Roberts 1995, Leasure et al. 2001, Gelatt 2003, Slatter 2005). Além disso, a escolha de cães idosos (média $=9,6$ anos) e diabéticos, que são mais susceptíveis a apresentarem doenças oftálmicas (Leasure et al. 2001) e sistêmicas (Basher \& Roberts 1995, Landry et al. 2004, Slatter 2005), poderiam comprometer as amostras. Portanto, o estabelecimento de um critério de exclusão rigoroso permitiu com que as amostras estudadas fossem livres de quaisquer alterações importantes (que não a diabete e a catarata), desde o dia do primeiro atendimento, até o dia da cirurgia. Entretanto, tais critérios acabaram por limitar de maneira importante o número de animais recrutados para o estudo, mas, por outro lado, os incluídos certamente garantiram a confiabilidade necessária para sua conclusão.

A realização da técnica cirúrgica de FACO em cataratas hipermaduras ainda é a melhor opção cirúrgica, já que a lente pode apresentar alterações significativas em sua estrutura capazes de comprometer o sucesso cirúrgico (Davidson et al. 1990). Venkatesh et al. (2010) observaram que a FACO em cataratas hipermaduras também são frequentemente realizadas em pacientes humanos atendidos na Índia, um país em desenvolvimento como o Brasil. Corroborando com Khanna et al. (2011), acreditamos que a justificativa de tal ocorrência, tanto pelos proprietários dos cães, quanto pelos pacientes humanos, seja o alto custo da cirurgia e a falta de profissionais habilitados nas diferentes regiões destes países.

As análises histoquímicas realizadas nas cápsulas colhidas neste estudo demonstraram que não houve diferença estatisticamente significante entre os grupos. Estudo realizado anteriormente em humanos evidenciou que há aumento na expressão de proteínas na membrana extracelular das cápsulas colhidas de pacientes diabéticos, quando comparados às colhidas de pacientes sadios que apresentavam apenas cataratas do tipo corticais, o que supostamente poderia alterar a composição dos proteoglicanos nessa estrutura e, por consequência, sua função nestes pacientes (Tiwari et al. 2014). Outros estudos referem que há uma maior produção de colágeno na presença de catarata e/ou diabete e que a alteração é ainda maior no grupo dos diabéticos, quando também comparados a pacientes sadios (Glover \& Constantinescu 1997, Bras et al. 2006, Pot et al. 2009, Wormstone et al. 2009, Chandler et al. 2012). Diferente dos referidos estudos, não se observou diferença significante quanto ao aumento na expressão de proteínas (proteoglicanos) e/ou colágeno entre os grupos estudados. Isto provavelmente deveu-se a todas as amostras pertencerem a grupos doentes, ou seja, supostamente capazes de gerar alterações significativas à estrutura.

\section{CONCLUSÕES}

Os resultados das análises realizadas mostraram que ambas as amostras, tanto as provenientes das cataratas hipermaduras, quanto das diabéticas, apresentam semelhante composição molecular de proteoglicanos e colágeno IV e isto independente da utilização de azul de tripano a 0,1\%.

Sugere-se a realização de novos estudos, com diferentes fases de evolução da catarata, idade de aparecimento e a realização de outras formas de análises laboratoriais, com diferentes concentrações de azul de tripano e intervalos de tempo de contato do corante com as amostras. Além disso, o estudo da composição molecular da cápsula anterior de um grupo controle contribuiria de forma significativa na avaliação e graduação da intensidade das lesões nos grupos futuramente estudados.

Agradecimentos.- À Coordenação de Aperfeiçoamento de Pessoal de Nível Superior-CAPES, pelo apoio à Pesquisa de Doutorado.

\section{REFERÊNCIAS}

Bailey A.J., Sims T.J., Avery N.C. \& Miles C.A. 1993. Chemistry of collagen cross-links: glucose-mediated covalent cross-linking of type-IV collagen in lens capsules. Biochem J. 296:489-496.

Barros L.F.M., Fernandes B.F., Bakalian S., Safatle A.M.V., Barros P.S.M. \& Burnier Jr M.N. 2008. Anterior lens capsule thickness in diabetic and non-diabetic dogs. ARVO Meeting Abstracts 49:2282.

Basher A.W. \& Roberts S.M. 1995. Ocular manifestations of diabetes mellitus: diabetic cataracts in dogs. Vet. Clin. North Am., Small Anim. Pract. 25(3):661-676.

Beam S., Correa M.T. \& Davidson M.G. 1999. A retrospective-cohort study on the development of cataracts in dogs with diabetes mellitus: 200 cases. Vet. Ophthalmol. 2:169-172.

Bernays M.E. \& Peiffer R.L. 2000. Morphologic alterations in the anterior lens capsule of canine eyes with cataracts. Am. J. Vet. Res. 61(12):1517-1519.

Bras D., Colitz C.M.H., Saville W.J.A., Gemensky-Metzler A.J. \& Wilkie D.A. 2006. Posterior capsular opacification in diabetic and non diabetic canine patients following cataract surgery. Vet. Ophthalmol. 9(5):317-327.

Chandler H.L., Haeussler Jr D.J., Gemensky-Metzler A.J., Wilkie D.A. \& Lutz E.A. 2012. Induction of posterior capsule opacification by hyaluronic acid in an ex vivo model. Invest. Ophthalmol. Vis. Sci. 53(4):1835-1845.

Chang Y.S., Tseng S.Y., Tseng S.H., Chen Y.T. \& Hsiao J.H. 2005. Comparison of dyes for cataract surgery. Part 1: cytotoxicity to corneal endothelial cells in a rabbit model. J. Cataract Refract. Surg. 31(4):792-798.

Danysh B.P. \& Duncan M.K. 2009. The lens capsule. Exp. Eye Res. 88(2): 151-164.

Davidson M.G., Nassisse M.P., Rusnak I.M., Corbett W.T. \& English R.V. 1990. Success Rates of Unilateral vs. Bilateral Cataract Extraction in Dogs. Vet. Surg. 19(3):232-236.

Davidson M.G. \& Nelms S.R. 2007. Diseases of the canine lens and cataract formation. In: Gelatt K.N. (Ed.), Veternary Ophthalmology. 4th ed. Ames, Iowa.

Dick H.B., Aliyeva S.E. \& Hengerer F. 2008. Effect of trypan blue on the elasticity of the human anterior lens capsule. J. Cataract Refract. Surg. 34:1367-1373.

Gelatt K.N. 2003. Manual de Oftalmologia Veterinária. 3ํㅗㄹ edição. Manole: São Paulo, 594p.

Gelatt K.N. \& Gelatt J.P. 2001. Surgical procedures of the lens and cataracts, p.305-355. In: Ibid. (Eds), Small animal ophthalmic surgery: a practical guide for the practicing veterinarian. Gainesville, Florida.

Glover T.D. \& Constantinescu G.M. 1997. Surgery for cataracts. Vet. Clin. North Am., Small Anim. Pract. 27(5):1143-1173.

Halfter W., Candiello J., Hu H., Zhang P., Schreiber E. \& Balasubramani M. 2013. Protein composition and biomechanical properties of in vivo-derived basement membranes. Cell Adh. Migr. 7(1):64-71. 
Jung Y., Kim M.S., Joo C. \& Chung S.K. 2013. Role of intrinsic apoptotic pathway in cataractogenesis in type 2 diabetic patients. J. Cataract Refract. Surg. 39:1470-1476.

Khanna R., Pujari S. \& Sangwan V. 2011. Cataract surgery in developing countries. Curr. Opin. Ophthalmol. 22(1):10-14.

Kim B., Kim S.Y. \& Chung S.K. 2012. Changes in apoptosis factors in lens epithelial cells of cataract patients with diabetes mellitus. J. Cataract Refract. Surg. 38:1376-1381.

Krag S. \& Andreassen T.T. 2003. Mechanical properties of the human posterior lens capsule. Invest Ophthalmol Vis. Sci. 44:691-96.

Landry M.P., Herring I.P. \& Panciera D.L. 2004. Fundoscopic findings following cataract extraction by means of phacoemulsification in diabetic dogs: 52 cases (1993-2003). J. Am. Vet. Med. Assoc. 225(5):709-716.

Leasure J., Gelatt K.N. \& Mackay E.O. 2001. The relationship of cataract maturity to intraocullar pressure in dogs. Vet. Ophthalmol. 4(4):273-276.

Lebleu V.S., Macdonald B. \& Kalluri R. 2007. Structure and Function of Basement Membranes. Mini review. Exp. Biol. Med. 232:1121-1129.

Marshall G.E., Konstas A.G.P., Bechrakis N.E. \& Lee W. 1992. An immune electronmicroscope study of the aged human lens capsule. Exp. Eye Res. 54:393-401.

Martin C.L. 2005. Vitreous and ocular fundus, p.401-460. In: Ibid. (Ed.), Ophthalmic Disease in Veterinary Medicine, London.

Mohamad N., Gutiérrez A., Núnez M., Cocca C., Martín G., Cricco G., Medina V., Rivera E. \& Bergoc R. 2005. Mitochondrial apoptotic pathways. Biocell 29:149-161.

Nanavaty M.A., Johar K., Sivasankaran M.A., Vasavada A.R., Praveen M.R. \& Zetterstro M.C. 2006. Effect of trypan blue staining on the density and viability of lens epithelial cells in white cataract. J. Cataract Refract. Surg. 32:1483-1488.

Nelson L.D. \& Cox M.M. 2011. Carboidratos e Glicobiologia, 236-270. In: Princípios da Bioquímica de Lehhinger. 5a ed. Sarvier Artmed.

Ofri R. 2008. Lens, p.258-276. In: Maggs D.J., Miller P.E. \& Ofri R.I. (Eds), Slatter's Fundamentals of Veterinary Ophthalmology. 4th ed. St Louis, Missouri.

Ozturk F. \& Osher R.H. 2006. Capsular staining: recent developments. Curr Opin. Ophthalmol. 17:42-44.

Portes A.L.F., Monteiro M.L.R., Allodi S. \& Miguel N.C.O. 2007. Effect of trypan blue on lens epithelial cells in human eyes having capsulorhexis. J. Cataract Refract. Surg. 33:1135.

Portes A.L.F., Almeida A.C., Allodi S., Monteiro M.L.R. \& Miguel N.C.O. 2010. Trypan blue staining for capsulorhexis: Ultrastructural effect on lens ep- ithelial cells and capsules. J. Cataract Refract. Surg. 36:582-587.

Pot S.A., Chandler H.L., Colita C.M.H., Bentley E., Dubielzig R.R. \& Mosley T.S., Reid T.W. \& Murphy C.J. 2009. Selenium functionalized intraocular lenses inhibit posterior opacification in an ex vivo canine lens capsular bag assay. Exp. Eye Res. 89:728-734.

Safatle A.M.V., Souza M.S.B., Teixeira R.B. \& Barros P.S.M. 2000. Catarata diabética com reabsorção em cães. Revta Bras. Ciênc. Vet. 7(Supl.):156.

Sigle K.J. \& Nassisse M.P. 2006. Long-term complications after phacoemulsification for cataract removal in dogs: 172 cases (1995-2002). J. Am. Vet. Med. Assoc. 228(1):74-79.

Slatter D. 2005. Fundamentos de Oftalmologia Veterinária. 3ํㅗ ed. Roca, São Paulo.

Smyth N., Vatansever H.S., Murray P., Meyer M., Frie C. \& Paulsson M. 1999. Absence of basement membranes after targeting the LAMC1 gene results in embryonic lethality due to failure of endoderm differentiation. J. Cell Biol. 144:151-160.

Tiwari A., Ram J. \& Luthra-Guptasarma M. 2014. Targeting the fibronectin type III in Tenascin-C inhibits epithelial mesenchymal transition in the context of Posterior Capsular Opacification. Invest. Ophthalmol. Vis. Sci. 56(1):272-283.

Vaughan D., Asbury T. \& Riordan-Eva P. 2003. Oftalmologia Geral. 15a ed. Atheneu, São Paulo. 432p.

Venkatesh R., Tan C.S., Sengupta S., Ravindran R.D., Krishnan K.T. \& Chang D.F. 2010. Phacoemulsification versus manual small-incision cataract surgery for white cataract. J. Cataract Refract. Surg. 36(11):1849-1854.

Voet D., Voet J.G. \& Pratt C.W. 2008. Carboidratos, p.219-224. In: Ibid. (Eds), Fundamentos da Bioquímica: a vida em nível molecular. $2^{\underline{a}}$ ed. Armed, São Paulo.

Winkler J., Wirbelauer C., Frank V. \& Laqua H. 2001. Quantitative distribution of glycosaminoglycans in young and senile (cataractous) anterior lens. Exp. Eye Res. 72:311-318.

Wollensak G., Sporl E. \& Pham D.T. 2003. Biomechanical changes in the anterior lens capsule after trypan blue staining. J. Cataract Refract. Surg. 30:1526-1530.

Wormstone M., Wang L. \& Liu C.S.C. 2009. Posterior capsule opacification. Exp Eye Res. 88:257-269.

Yan Q., Perdue N., Blake D. \& Sage E.H. 2005. Absence of SPARC in murine lens epithelium leads to increased exposition of laminin-1 in lens capsule. Invest. Ophthalmol. Vis. Sci. 46(12):4652-4660.

Yang X., Zou L., Binrong M., Dong D., Dai H. \& Lu X. 1998. Tensile strength of lens capsules in eye-bank eyes. J. Cataract Refract. Surg. 24:543-546. 